\title{
Pedicled temporoparietal fascial flap for combined revascularization in adult moyamoya disease
}

\author{
Kristine Ravina, MD, ${ }^{1}$ Robert C. Rennert, MD, ${ }^{2}$ Ben A. Strickland, MD, ${ }^{3}$ Mark Chien, BA, ${ }^{1}$ \\ Joseph N. Carey, MD, ${ }^{4}$ and Jonathan J. Russin, MD ${ }^{1,3}$
}

${ }^{1}$ Neurorestoration Center, ${ }^{3}$ Department of Neurological Surgery, and 4Division of Plastic and Reconstructive Surgery, Department of Surgery, Keck School of Medicine, University of Southern California, Los Angeles; and 'Department of Neurosurgery, University of California, San Diego, California

\begin{abstract}
Moyamoya disease (MMD) is a progressive, idiopathic cerebrovascular occlusive disease. Various revascularization techniques including direct, indirect, and combined microvascular bypasses have been described. This article presents a modified revascularization technique for MMD utilizing a pedicled temporoparietal fascial flap (TPFF) for combined revascularization. This technique combines a large area of coverage for indirect revascularization with the benefits of a direct bypass. The pedicled TPFF also benefits from intact venous drainage to minimize the risk of flap swelling that could result in complications from mass effect.
\end{abstract}

https://thejns.org/doi/abs/10.3171/2018.5.JNS18938

KEYWORDS cerebral revascularization; combined bypass surgery; STA-MCA bypass; moyamoya disease; temporoparietal pedicled flap; surgical technique; vascular disorders

$\mathrm{M}$ OYAMOYA disease (MMD) is an idiopathic cerebrovascular disease characterized by progressive occlusion of the internal carotid artery (ICA) terminus and its main branches, followed by abnormal collateral vascular network development. ${ }^{22,31,33}$ A typical presentation of MMD is ischemic or hemorrhagic stroke that tends to recur along the natural course of MMD, resulting in neurocognitive decline and functional deficits. . $^{5,31,33}$ Currently, surgical revascularization is recognized as an effective treatment option for MMD patients. ${ }^{22,31,39}$ Revascularization techniques are classified as direct, indirect, or combined. ${ }^{4,18,22,31}$ Since the first direct superficial temporal artery (STA)-to-middle cerebral artery (MCA) bypass was performed by Yaşargil in 1967, this approach has been widely used with good results for ischemic cerebrovascular pathologies. ${ }^{18,26,37}$ STA-MCA bypass has been proven effective for reduction of ischemic as well as hemorrhagic recurrences in MMD. It has thus become the treatment of choice for adult MMD at most institutions..$^{12,16,23,26,27}$ In addition to direct revascularization, which involves extracranial-to-intracranial (EC-IC) microvascular anastomosis, indirect approaches like encephalo-duro-arterio-synangiosis provide an opportunity to supplement collateral blood flow by neovascularization. In pediatric patients, the relatively small vessel diameter renders microvascular anastomosis particularly challenging. Additionally, indirect bypass results in a more robust potential for neovascularization, being preferred in pediatric MMD. 1,9,15,22,25,27

The use of the temporoparietal fascial flap (TPFF) in reconstructive plastic surgery was described more than a century ago. ${ }^{28}$ The reliability of the TPFF as a highly vascular flap has expanded its application as a versatile pedicled as well as a free and composite flap.,11,28 While successful use of a TPFF for indirect revascularization in pediatric MMD has been previously described, it has never been applied for combined revascularization in adults.

ABBREVIATIONS EC-IC = extracranial-to-intracranial; ICA = internal carotid artery; MCA = middle cerebral artery; MMD = moyamoya disease; STA = superficial temporal artery; STV = superficial temporal vein; TPF = temporoparietal fascia; TPFF = temporoparietal fascial flap.

SUBMITTED April 5, 2018. ACCEPTED May 29, 2018.

INCLUDE WHEN CITING Published online November 9, 2018; DOI: 10.3171/2018.5.JNS18938. 
The isolated use of indirect revascularization does not provide immediate blood flow supplementation, has been associated with less favorable outcomes, and is typically not considered the treatment of choice in adult MMD. ${ }^{12,22 \text {, }}$ $23,32,35,39$

Here, we present a novel revascularization technique combining direct STA-MCA bypass with indirect revascularization through a pedicled TPFF. Besides direct cerebral blood flow augmentation from the STA, this technique offers supplemented collateral neovascularization from a large surface area of a highly vascular TPFF placed in direct contact with the brain surface. A detailed description of the technique along with a clinical case is presented.

\section{Methods}

Patients with MMD are considered for this approach of combined revascularization if they are symptomatic or present with clinically silent ischemia on imaging in addition to the presence of donor and recipient vessels suitable for anastomosis based on angiography findings.

\section{Anatomy and Nomenclature}

The temporoparietal fascia (TPF), also known as superficial temporal fascia, epicranial aponeurosis, and galeal extension, is a thin connective tissue layer located directly underneath the hair follicles and fibrofatty subdermal tissue. ${ }^{20,36}$ At the level of the superior temporal line, the TPF is continuous with the galea aponeurotica. The TPF is continuous with the subcutaneous musculoaponeurotic system of the face. Anteriorly and posteriorly, the TPF is attached to the frontalis and occipitalis muscles, respectively. The deep temporal fascia is continuous with the pericranium superiorly, directly covers the temporalis muscle, and is separated from the TPF above by a loose areolar tissue layer. ${ }^{2,10,20,29,36}$ The TPF is supplied by the STA, which courses through it. TPF drainage is provided by the superficial temporal vein (STV) located slightly superficial to the STA. ${ }^{20,36}$

\section{Surgical Technique}

The patient is placed in a supine position, and the head is secured in a skull clamp, turned almost completely laterally. A slight tilt is maintained to facilitate the runoff of irrigation during the dissection and anastomosis.

\section{Harvesting of the TPFF}

The course of the STA and its branches (Fig. 1) are mapped using Doppler ultrasonography, and their location is marked on the scalp (see Video 1 for the surgical summary).

VIDEO 1. Three-dimensional operative video of an example case demonstrating use of pedicled TPFF in combined revascularization with STA-MCA bypass. Copyright Jonathan J. Russin. Published with permission. Click here to view.

Subsequently, a Y-shaped incision is performed originating in the periauricular crease, starting proximally at the level of the tragus and extending into a Y shape at the level of the superior temporal line to maximize galeal exposure.
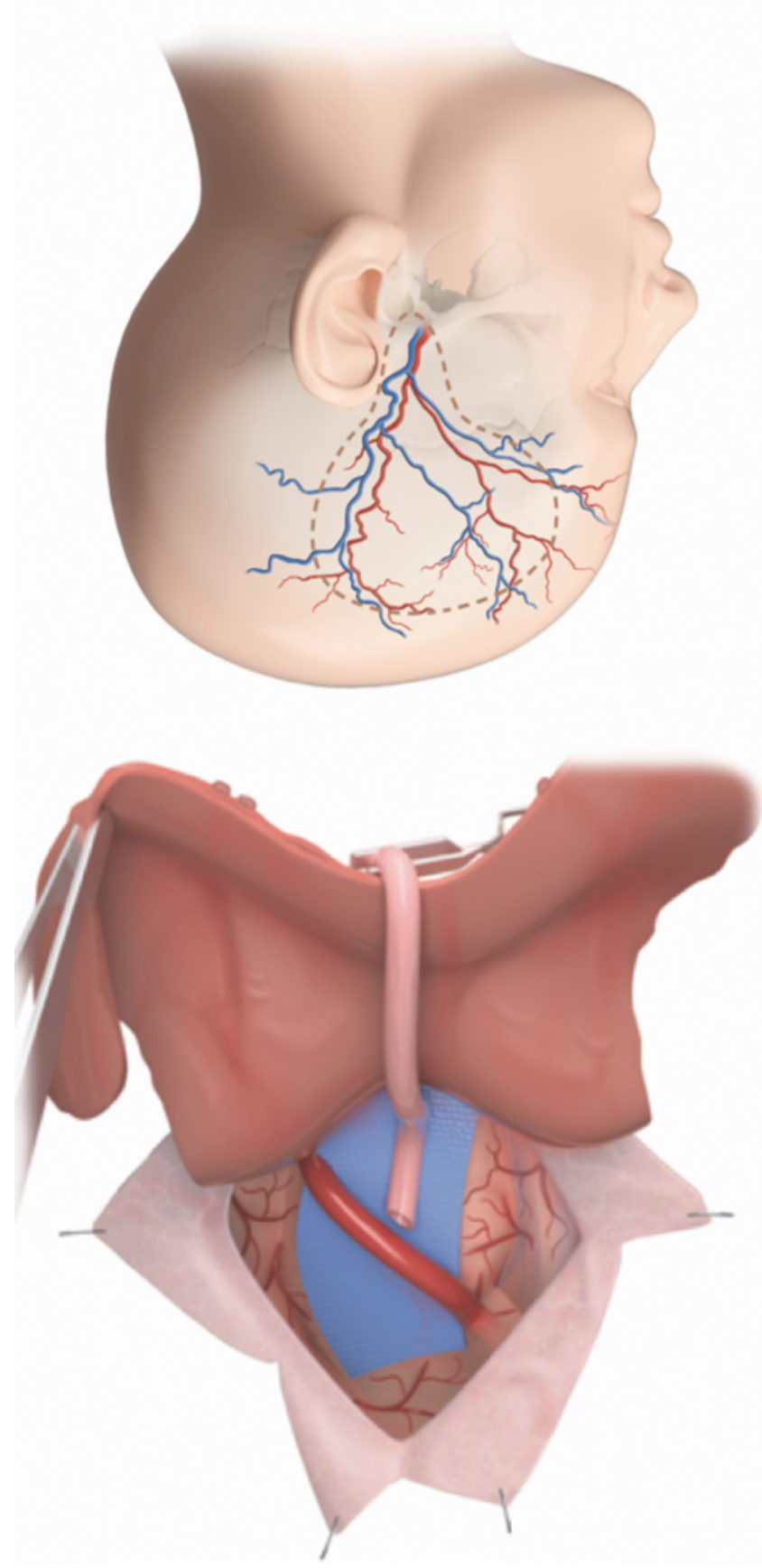

FIG. 1. Outline of the TPFF location and a depiction of the butterflying step of the modified combined STA-MCA and TPFF bypass procedure. Upper: The extent of the TPFF harvested for the combined bypass is outlined (dashed line) relative to the anatomical landmarks, STA, and STV as well as their frontal and parietal branches. Lower: Exposure of the donor and recipient vessel after making a cut in the TPFF and butterflying the distal half of the flap to allow passage of the STA to the cortical anastomosis site. Copyright Jonathan J. Russin. Published with permission.

The skin is gently elevated in the adipocutaneous plane, taking care to maintain integrity of the hair follicles for long-term alopecia prevention. Wide-angled scalp flaps $\left(>45^{\circ}\right)$ are recommended to avoid ischemia of the wound 
edges. ${ }^{38}$ Other types of incisions, such as lazy $\mathrm{S}$ and a preauricular linear incision with a coronal extension up to the midline, have been described. ${ }^{28,30}$

After maximizing the exposure of the TPFF, a proximal dissection is performed to define an approximately $1-\mathrm{cm}$ pedicle including the STA and the STV. Subsequent dissection is performed to raise an approximately $8 \times 6-\mathrm{cm}$ fascial flap off of the superficial layer of the deep temporal fascia and the pericranium. While performing the anterior flap elevation, attention should be paid to the TPFF's relationship to the frontal and temporal branches of the facial nerve. ${ }^{11}$ Raising the TPFF on the parietal branch of the STA when suitable can help to avoid nerve injury. ${ }^{10}$ Typically, the parietal branch of the STA is identified and circumferentially dissected from its most distal exposure proximally to the middle of the flap. A temporary clip is used to occlude the dissected branch at its most proximal dissection point. The vessel is then divided at a point to maximize its length and is flushed with heparinized saline. The TPFF is mobilized inferiorly and wrapped in a nonstick gauze pledget soaked in a heparin and milrinone solution.

\section{Direct STA-MCA Bypass and Indirect Revascularization Through TPFF}

The temporalis fascia and muscle are divided using monopolar cautery making a T-shaped incision to facilitate exposure. A subperiostal dissection is performed to elevate the muscular flaps, which are then retracted. A standard frontotemporal craniotomy is performed, and the bone flap is elevated. At the inferior margin, a craniectomy is performed with a coarse diamond drill to facilitate the TPFF pedicle. A durotomy is performed, taking great care to preserve the main branches of the middle meningeal artery, as it has been shown to be an important source of collateral neovascularization. ${ }^{21}$ The resulting dural flaps are reflected using 4-0 sutures. A sufficient cortical MCA branch is identified and circumferentially dissected. The TPFF is mobilized intradurally, and a cut is made in the TPFF "butterflying" the distal half of the flap to allow passage of the STA to the cortical anastomosis site underneath the flap (Fig. 1). Subsequently, the STA is "fishmouthed" and approximated to the recipient vessel. The "butterfly wings" of the flap are reflected up to the surrounding temporalis muscle using staples or suture to maintain visualization during the anastomosis.

The patient is placed in burst suppression, and a standard end-to-side microvascular anastomosis is performed using 10-0 nylon suture. After inspection of the anastomosis, temporary clips are removed from the STA, and indocyanine green dye fluorescence is used to evaluate the patency of the anastomosis.

The wings of the TPFF flap are released, and the flap is laid directly over the cortical surface (Fig. 2). The flap is typically larger than the craniotomy, and the remainder is tucked under the edges of the exposure. The flap is tacked to the dura using 4-0 silk suture. The dural leaflets are laid over the TPFF and loosely reapproximated using 4-0 sutures. The bone is fixed in place using cranial plates (Fig. 2), and the temporalis fascia is reapproximated using interrupted 2-0 sutures, allowing the TPFF pedicle to pass without compression. Previously elevated scalp flaps are remobilized toward the midline; 4-0 deep dermal sutures are placed in order to maximize dermal closure and to protect hair follicles. The skin is then closed using 5-0 suture.

\section{Case Presentation}

A 29-year-old woman presented with acute left hemi-
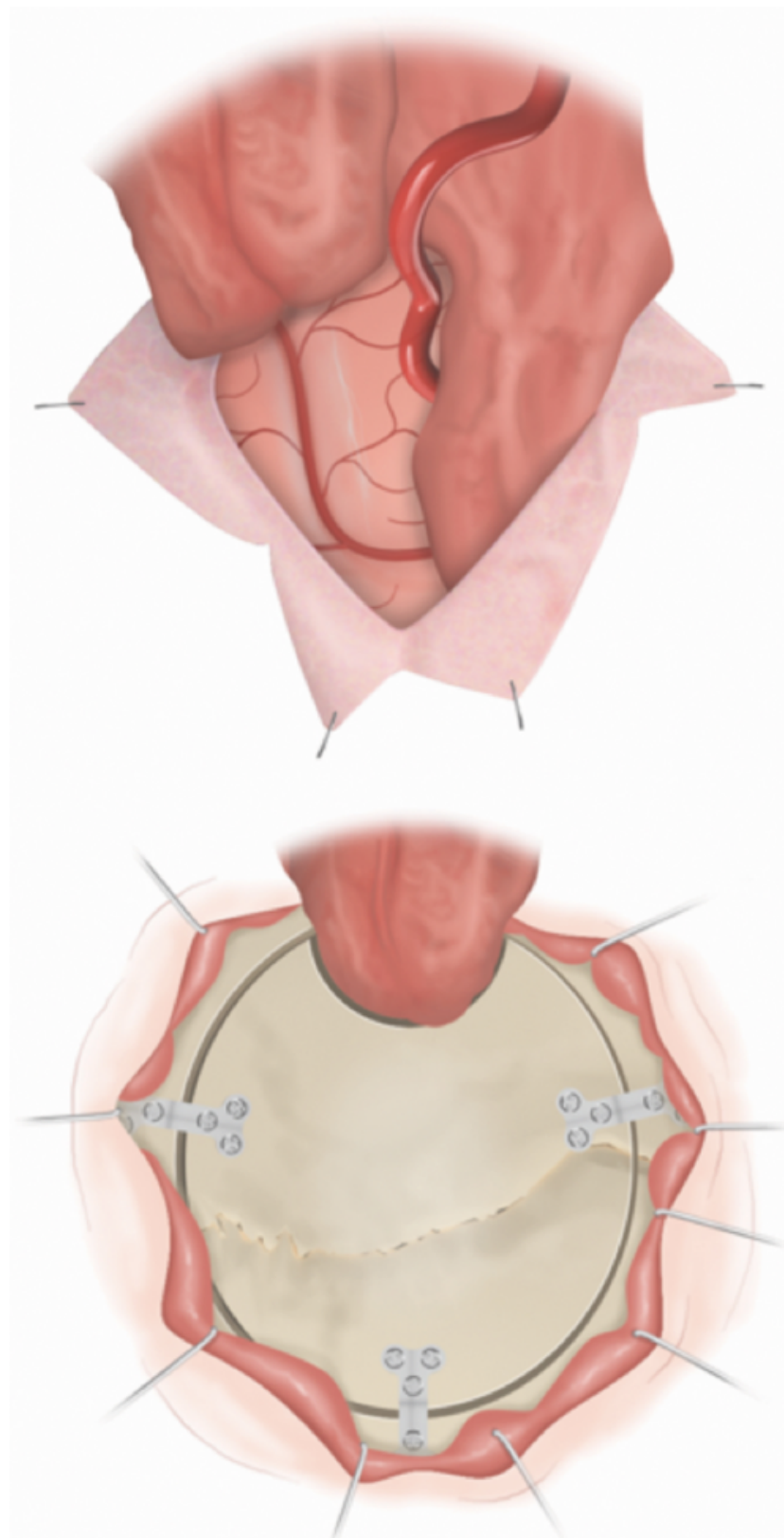

FIG. 2. Schematic illustration of the TPFF intradural positioning and replantation of the bone flap. Upper: TPFF butterfly wings are released and relocated in direct contact with the brain surface for indirect revascularization. The edges of the TPFF are tucked under the craniotomy margins. Lower: The bone flap is repositioned and fixed in place using cranial plates. The opening in the bone flap has been tailored using a rongeur, allowing the TPFF pedicle to pass through freely. Copyright Jonathan J. Russin. Published with permission. 


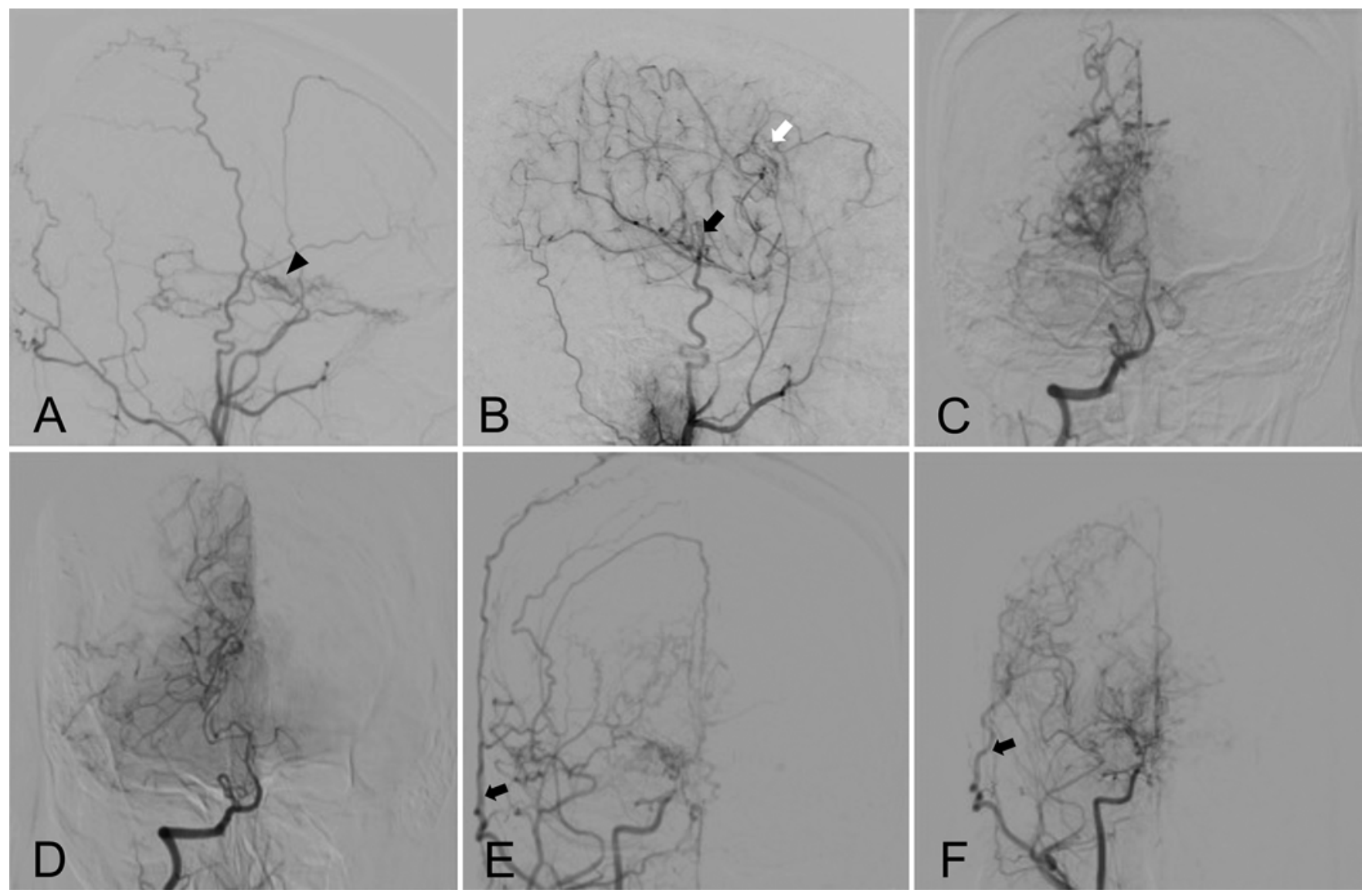

FIG. 3. Preoperative diagnostic and 15 -month postsurgery follow-up cerebral angiograms. A: Preoperative right ICA and external carotid artery (ECA) injection demonstrating moyamoya vasculature at the ICA terminus (arrowhead). B: Follow-up right ECA injection demonstrating STA-MCA anastomosis (black arrow). Neovascularization from the TPFF results in a hazy appearance (white arrow). C: Preoperative right vertebral artery injection demonstrating pathological moyamoya vessels notable at the basilar terminus and posterior cerebral artery territory. D: Follow-up right vertebral artery injection showing reduction of pathologic moyamoya vasculature. E: Right ECA injection showing a robust STA (black arrow). F: Follow-up right ECA injection showing robust flow in the STA passing into the intracranial space (black arrow) and evidence of neovascularization from the bypass.

paresis and was found to have a small right thalamic hemorrhage. Initial preoperative CT angiography demonstrated pathological vascular changes consistent with bilateral MMD. Subsequent catheter angiography (Fig. 3A, C, and E) revealed significant stenosis of the distal right ICA and very little filling of right MCA from the ICA injection (Fig. $3 A)$. The right posterior MCA territory filled predominantly via leptomeningeal collaterals from the right posterior cerebral artery. Moyamoya vessels were identified at the basilar and carotid termini bilaterally (Fig. 3A and C). The patient underwent a right combined revascularization with a pedicled TPFF as described above. Intraoperative indocyanine green imaging and postoperative CT angiography revealed good patency of the STA-MCA anastomosis. The patient's postoperative neurological examination findings were stable, and she was ultimately transferred for inpatient rehabilitation. Follow-up at 10 months post-bypass surgery showed improved left-sided strength with no new or worsening symptoms. MRI displayed no evidence of new hemorrhagic or ischemic complications. A patent direct bypass and evidence of indirect revascularization from the TPFF were appreciated on follow-up MR angi- ography. The patient was residing at home and performing all activities of daily living independently. Her wound had healed well with minimal postoperative alopecia. Followup catheter angiography performed at 15 months post-bypass surgery also showed a patent STA-MCA bypass with robust flow in sylvian vessels with reduction of moyamoya pathological vasculature (Fig. 3B, D, and F).

\section{Discussion}

Surgical treatment for MMD is aimed at providing an EC-IC bypass for cerebral blood flow supplementation to ischemic brain regions. A variety of surgical revascularization techniques are available for adult MMD patients and are generally divided into 3 categories: direct, indirect, and combined bypass. These approaches include the use of a donor artery (STA, middle meningeal artery, or deep temporal artery) and/or highly vascular tissue (temporalis muscle, dura mater, or omentum) placed in direct contact with the brain surface. ${ }^{4,15,18}$

Direct revascularization provides the benefit of immediate and generally reliable blood flow supplementa- 
tion. Possible drawbacks of direct revascularization include the need for matching donor and recipient artery calibers, technical challenges, hyperperfusion syndrome, and stroke. ${ }^{1,15,17,23}$ Indirect revascularization is considered technically easier and faster than direct revascularization, but it relies on late and slow revascularization, which can take up to several months to develop. ${ }^{1,14,15,23,39}$ Isolated indirect bypass has been described as a successful and sufficient method for pediatric MMD, as pediatric patients have demonstrated stronger angioplasticity potential and response over time. . $^{8,915,17,18,34,39}$

Thus far, there have been reports of combined revascularization having at least the same capacity as that of isolated direct bypass for the reduction of postoperative stroke. ${ }^{8,9,18,23}$ This is supported by a recent extensive metaanalysis by Sun et al. of 47 studies with a total of 2013 adult MMD patients. In this analysis, it was concluded that direct and combined bypasses result in comparable risk reduction of long-term complications that is superior to that achieved by isolated indirect bypass. ${ }^{32}$ In addition, MMD patients treated with combined bypass have shown a trend toward better postoperative angiographic revascularization outcomes and more prominent cerebral blood flow improvement than those treated with isolated indirect or direct bypass. ${ }^{6,18,19}$ Noteworthy, in combined approaches, patency of the direct bypass can have either an inverse or a complementary relation to the extent of neovascularization from indirect bypass depending on demand for supplemental blood flow. This supports the concept of the revascularization graft as a dynamic, selfremodeling structure. ${ }^{3,15}$ In this context, use of a combined approach is advantageous, as it provides an additional option for neovascularization if the direct bypass fails or becomes insufficient.

To date, there have not been any randomized, controlled trials comparing surgical revascularization approaches for MMD. Interestingly, there are studies reporting worse postoperative outcomes in adult MMD patients treated with direct or combined bypass compared with an indirect approach. ${ }^{17,24,34}$ One potential explanation for conflicting results may be the presence of a selection bias for the type of revascularization performed in certain clinical scenarios. For example, clinically more advanced and complicated MMD bears a higher risk for negative outcome, and the choice of direct revascularization is favored over indirect revascularization, knowing the limited ability of indirect bypass to immediately reduce ischemia risk. Other known limitations associated with comparison studies of MMD revascularization approaches include small sample size, high variability in patient population, and surgical approach preferences of individual centers and surgeons. While these limitations might prevent results from reaching statistical significance, combined revascularization is commonly being recommended as the treatment of choice for adult MMD. ${ }^{6,8,18,19}$

The combined revascularization technique reported here presents the use of direct STA-MCA bypass with indirect bypass through a TPFF. The TPFF is a highly vascular lateral extension of the galea supplied by the STA and located directly underneath the subcutaneous fat layer. ${ }^{10}$ Use of the TPFF as a pedicled graft is an advanta- geous and time-efficient approach due to its anatomical location and the fact that only one anastomosis is required. The most common complication reported in association with TPFF harvest is alopecia due to the proximity of hair follicles, which can be easily damaged during dissection. ${ }^{11}$ Nevertheless, as demonstrated by outcomes published by other authors, this complication can be mitigated. ${ }^{28,29}$ Other complications are also possible. After harvest of the TPFF, there is a relatively thin scalp layer remaining for closure. Additionally, the trifurcation from the Y-shaped incision creates a risk for ischemic wound complications. The current reported rate of wound dehiscence is approximately $8 \%$ or less; however, the cohorts tend to be relatively small. ${ }^{13,28}$ During dissection, efforts should be made to not compromise the other STA branch not used in direct revascularization whenever possible, as this can heighten the risk of ischemic wound complications. If an acute, symptomatic direct bypass failure occurs in this situation requiring an alternative direct revascularization, the remaining STA stump or another extracranial donor, such as the internal maxillary or facial artery, can then be used with an interposition graft like the descending branch of the lateral circumflex femoral artery or radial artery.

TPFF use in combined revascularization offers several potential benefits over previously described, traditional combined revascularization techniques. First, a sizable, highly vascular fascial flap is used, which does not require the inversion needed in approaches utilizing dura like encephalo-duro-synangiosis and encephalo-duroarterio-synangiosis. Second, it offers large brain surface area coverage for neovascularization without significant mass effect, which is a concern in approaches utilizing the temporalis muscle. In addition, avoiding the use of the temporalis muscle eliminates the risk of myoelectric activity-induced epileptic seizures and offers better cosmetic and functional results. ${ }^{35}$ Finally, the vascular pedicle of the flap allows preservation of the STV, which helps to prevent flap venous engorgement and swelling. Despite these potential benefits, sufficiently sized cohort studies of long-term outcomes in adult MMD patients treated with this novel combined approach are certainly necessary to assess the benefit and complication rates.

\section{Conclusions}

While there is ongoing controversy regarding the most effective revascularization approach in MMD, a growing pool of evidence supports direct and combined revascularization in adult patients. The technique of combined revascularization presented here has not previously been described. The perceived benefit over current revascularization strategies is the extent of coverage using a local, well-perfused vascular flap with preserved venous outflow that can result in acceptable cosmetic outcomes. More extensive evaluation of this technique will be required to discern its value in the treatment of MMD.

\section{References}

1. Abla AA, Gandhoke G, Clark JC, Oppenlander ME, Velat GJ, Zabramski JM, et al: Surgical outcomes for moyamoya angiopathy at barrow neurological institute with comparison 
of adult indirect encephaloduroarteriosynangiosis bypass, adult direct superficial temporal artery-to-middle cerebral artery bypass, and pediatric bypass: 154 revascularization surgeries in 140 affected hemispheres. Neurosurgery 73:430-439, 2013

2. Allegretti JP, Panje WR: The temporoparietal fascial flap in head and neck reconstruction: the workhorse from above. Oper Tech Otolaryngol Head Neck Surg 11:143-146, 2000

3. Amin-Hanjani S, Singh A, Rifai H, Thulborn KR, Alaraj A, Aletich V, et al: Combined direct and indirect bypass for moyamoya: quantitative assessment of direct bypass flow over time. Neurosurgery 73:962-968, 2013

4. Arias EJ, Derdeyn CP, Dacey RG Jr, Zipfel GJ: Advances and surgical considerations in the treatment of moyamoya disease. Neurosurgery 74 (Suppl 1):S116-S125, 2014

5. Calviere L, Catalaa I, Marlats F, Viguier A, Bonneville F, Cognard C, et al: Correlation between cognitive impairment and cerebral hemodynamic disturbances on perfusion magnetic resonance imaging in European adults with moyamoya disease. Clinical article. J Neurosurg 113:753-759, 2010

6. Choi IJ, Cho SJ, Chang JC, Park SQ, Park HK: Angiographic results of indirect and combined bypass surgery for adult moyamoya disease. J Cerebrovasc Endovasc Neurosurg 14:216-222, 2012

7. Collar RM, Zopf D, Brown D, Fung K, Kim J: The versatility of the temporoparietal fascia flap in head and neck reconstruction. J Plast Reconstr Aesthet Surg 65:141-148, 2012

8. Czabanka M, Peña-Tapia P, Scharf J, Schubert GA, Münch E, Horn P, et al: Characterization of direct and indirect cerebral revascularization for the treatment of European patients with moyamoya disease. Cerebrovasc Dis 32:361-369, 2011

9. Czabanka M, Vajkoczy P, Schmiedek P, Horn P: Age-dependent revascularization patterns in the treatment of moyamoya disease in a European patient population. Neurosurg Focus 26(4):E9, 2009

10. David SK, Cheney ML: An anatomic study of the temporoparietal fascial flap. Arch Otolaryngol Head Neck Surg 121:1153-1156, 1995

11. Demirdover C, Sahin B, Vayvada H, Oztan HY: The versatile use of temporoparietal fascial flap. Int J Med Sci 8:362-368, 2011

12. Deng X, Gao F, Zhang D, Zhang Y, Wang R, Wang S, et al: Direct versus indirect bypasses for adult ischemic-type moyamoya disease: a propensity score-matched analysis. J Neurosurg 128:1785-1791, 2018

13. Higgins KM, Ashford B, Erovic BM, Yoo J, Enepekides DJ: Temporoparietal fascia free flap for pharyngeal coverage after salvage total laryngectomy. Laryngoscope 122:523-527, 2012

14. Houkin K, Kamiyama H, Takahashi A, Kuroda S, Abe H: Combined revascularization surgery for childhood moyamoya disease: STA-MCA and encephalo-duro-arterio-myosynangiosis. Childs Nerv Syst 13:24-29, 1997

15. Houkin K, Kuroda S, Ishikawa T, Abe H: Neovascularization (angiogenesis) after revascularization in moyamoya disease. Which technique is most useful for moyamoya disease? Acta Neurochir (Wien) 142:269-276, 2000

16. Kawaguchi S, Okuno S, Sakaki T: Effect of direct arterial bypass on the prevention of future stroke in patients with the hemorrhagic variety of moyamoya disease. J Neurosurg 93:397-401, 2000

17. Kazumata K, Ito M, Tokairin K, Ito Y, Houkin K, Nakayama $\mathrm{N}$, et al: The frequency of postoperative stroke in moyamoya disease following combined revascularization: a single-university series and systematic review. J Neurosurg 121:432440, 2014

18. Kim DS, Huh PW, Kim HS, Kim IS, Choi S, Mok JH, et al: Surgical treatment of moyamoya disease in adults: combined direct and indirect vs. indirect bypass surgery. Neurol Med Chir (Tokyo) 52:333-338, 2012

19. Kim DS, Yoo DS, Huh PW, Kang SG, Cho KS, Kim MC: Combined direct anastomosis and encephaloduroarteriogaleosynangiosis using inverted superficial temporal artery-galeal flap and superficial temporal artery-galeal pedicle in adult moyamoya disease. Surg Neurol 66:389-395, 2006

20. Kim JC, Hadlock T, Varvares MA, Cheney ML: Hair-bearing temporoparietal fascial flap reconstruction of upper lip and scalp defects. Arch Facial Plast Surg 3:170-177, 2001

21. Komura S, Mikami T, Sugino T, Suzuki Y, Komatsu K, Wanibuchi M, et al: Complementary relation between direct and indirect bypass in progress of collateral circulation in moyamoya disease. World Neurosurg 104:197-204, 2017

22. Kuroda S, Houkin K: Moyamoya disease: current concepts and future perspectives. Lancet Neurol 7:1056-1066, 2008

23. Lee SB, Kim DS, Huh PW, Yoo DS, Lee TG, Cho KS: Longterm follow-up results in 142 adult patients with moyamoya disease according to management modality. Acta Neurochir (Wien) 154:1179-1187, 2012

24. Macyszyn L, Attiah M, Ma TS, Ali Z, Faught R, Hossain A, et al: Direct versus indirect revascularization procedures for moyamoya disease: a comparative effectiveness study. J Neurosurg 126:1523-1529, 2017

25. Matsushima T, Inoue TK, Suzuki SO, Inoue T, Ikezaki K, Fukui M, et al: Surgical techniques and the results of a fronto-temporo-parietal combined indirect bypass procedure for children with moyamoya disease: a comparison with the results of encephalo-duro-arterio-synangiosis alone. Clin Neurol Neurosurg 99 (Suppl 2):S123-S127, 1997

26. Miyamoto S, Yoshimoto T, Hashimoto N, Okada Y, Tsuji I, Tominaga T, et al: Effects of extracranial-intracranial bypass for patients with hemorrhagic moyamoya disease: results of the Japan Adult Moyamoya Trial. Stroke 45:1415-1421, 2014

27. Mizoi K, Kayama T, Yoshimoto T, Nagamine Y: Indirect revascularization for moyamoya disease: is there a beneficial effect for adult patients? Surg Neurol 45:541-549, 1996

28. Mokal NJ, Ghalme AN, Kothari DS, Desai M: The use of the temporoparietal fascia flap in various clinical scenarios: a review of 71 cases. Indian J Plast Surg 46:493-501, 2013

29. Nayak VK, Deschler DG: Pedicled temporoparietal fascial flap reconstruction of select intraoral defects. Laryngoscope 114:1545-1548, 2004

30. Pinto FR, de Magalhães RP, de Aquino Capelli F, Brandão LG, Kanda JL: Pedicled temporoparietal galeal flap for reconstruction of intraoral defects. Ann Otol Rhinol Laryngol 117:581-586, 2008

31. Research Committee on the Pathology and Treatment of Spontaneous Occlusion of the Circle of Willis: Guidelines for diagnosis and treatment of moyamoya disease (spontaneous occlusion of the circle of Willis). Neurol Med Chir (Tokyo) 52:245-266, 2012

32. Sun H, Wilson C, Ozpinar A, Safavi-Abbasi S, Zhao Y, Nakaji P, et al: Perioperative complications and long-term outcomes after bypasses in adults with moyamoya disease: a systematic review and meta-analysis. World Neurosurg 92:179-188, 2016

33. Suzuki J, Takaku A: Cerebrovascular "moyamoya" disease. Disease showing abnormal net-like vessels in base of brain. Arch Neurol 20:288-299, 1969

34. Teo MK, Madhugiri VS, Steinberg GK: Editorial. Direct versus indirect bypass for moyamoya disease: ongoing controversy. J Neurosurg 126:1520-1522, 2017

35. Wong WW, Hiersche MA, Zouros A, Martin MC: Indirect cerebral revascularization with a temporoparietal fascial flap in pediatric moyamoya patients: a novel technique and review of current surgical options. J Craniofac Surg 24:2039-2043, 2013 
36. Wormald PJ, Alun-Jones T: Anatomy of the temporalis fascia. J Laryngol Otol 105:522-524, 1991

37. Yasargil MG, Yonekawa Y: Results of microsurgical extraintracranial arterial bypass in the treatment of cerebral ischemia. Neurosurgery 1:22-24, 1977

38. Yokoyama R, Mikami T, Ukai R, Komatsu K, Kimura Y, Suzuki $\mathrm{H}$, et al: Interdisciplinary prevention and management of wound-related complications in extracranial-to-intracranial bypass surgery. World Neurosurg 115:247-253, 2018

39. Zipfel GJ, Fox DJ Jr, Rivet DJ: Moyamoya disease in adults: the role of cerebral revascularization. Skull Base 15:27-41, 2005

\section{Disclosures}

The authors report no conflict of interest concerning the materials or methods used in this study or the findings specified in this paper.

\section{Author Contributions}

Conception and design: Russin, Ravina, Rennert, Strickland. Acquisition of data: Russin, Ravina, Carey. Analysis and interpretation of data: Russin, Ravina. Drafting the article: Ravina. Critically revising the article: Russin, Ravina, Rennert. Reviewed submitted version of manuscript: all authors. Administrative/technical/material support: Russin, Chien, Carey. Study supervision: Russin.

\section{Supplemental Information}

Videos

Video 1. https://vimeo.com/280195136.

\section{Correspondence}

Jonathan J. Russin: The Keck School of Medicine, University of Southern California, Los Angeles, CA. jonathan.russin@med. usc.edu. 